\title{
The best-approximate realization of a spatial stiffness matrix with simple springs connected in parallel
}

\author{
Jue YU, Yong ZHAO*, Hao WANG, Genliang CHEN, Xinmin LAI \\ State Key Laboratory of Mechanical System and Vibration, Shanghai Jiao Tong University, \\ Shanghai Key Laboratory of Digital Manufacture for Thin-walled Structures, Shanghai Jiao Tong University, \\ 800 Dongchuan RD, Minhang District, Shanghai, China \\ *E-mail: zhaoyong@sjtu.edu.cn; Tel: +86-13761981187
}

\begin{abstract}
This paper presents an approximation algorithm to simplify the physical implementation of the non-isotropic stiffness matrices. The algorithm is inspired by the best approximation theorem in Matrix theory, by which the original stiffness matrix is projected onto a special linear space, thus an approximate isotropic matrix is derived. Accordingly, the desired force-deflection characteristics can be realized by purely simple springs (linear/torsional springs) connected in parallel. In mathematics, the approximation only changes the diagonal entries of the off-diagonal blocks of the original stiffness matrix. Moreover, the physical properties of the approximate isotropic matrix are identical to that of the original stiffness matrix in many aspects, including the center of stiffness, the wrench compliance axes as well as the translational and rotational stiffness value. The approximation is unique in both mathematics and physics. In order to illustrate the effectiveness of the proposed method, a numerical example is studied with comparison to some traditional algorithms of stiffness decomposition. The advantages of the approximation are well verified through the example.
\end{abstract}

Keywords: Stiffness matrix, best approximation, simple springs, force-deflection characteristics, isotropic.

\section{Introduction}

Appropriate mechanical interaction between the manipulator and circumstances is one of the most important problems in the use of robots in manufacturing tasks such as material removal and automated assembly. Without some form of force regulation, excessive interaction forces may arise from small manipulator positional misalignment and then lead to the failure of task [1]. To avoid damage to both the manipulator and the environments, the passive compliance which can be 
modeled as a kinematically unconstrained rigid-body suspended by several elastic units is generally used to provide some degrees of compliance at the end effector of a robot. The typical example of such a device is the Remote Center Compliance (RCC) device [2]. Proper design and use of such devices can result in a significant improvement in the reliability of manufacturing tasks [3]. In mathematics, the compliance/stiffness requirements can be quantized by a $6 \times 6$ stiffness matrix. As a result, the design of passive compliance can be transformed as a decomposition of the desired stiffness matrix. The decomposition will produce several spring wrenches. The wrench determines the placement and type of the passive spring that connects the suspended rigid-body and base of the passive compliance device. Many researchers have made contributions to the synthesis and realization of desired spatial stiffness matrix. Notably, Loncaric [4] pioneered an algorithm to synthesize a realizable spatial stiffness matrix with no more than 20 linear springs. Then the number of required simple springs for synthesizing the realizable stiffness matrix was reduced to seven at most by Huang and Schimmels [5]. The two scholars [6] also proposed the eigenscrew decomposition which is invariant in the coordinate transformation. Lipkin and Patterson $[7,8]$ presented the concept of wrench compliance axis and its dual, namely twist compliance axis. The former refers to a wrench along the axis yields a parallel translational deflection, and the latter implies a twist deflection along the axis causes a parallel reacting couple. The results were adopted to geometrically decompose the stiffness matrices into two sets of orthogonal spring wrenches with finite and infinite pitches $[8,9]$. In order to reduce the complexity of the compliance device, the minimal realization of the desired stiffness matrix was put forward by Roberts $[3,10]$ to minimize the number of passive springs. In addition, $\mathrm{Li}$ [11] researched the realization of an isotropic stiffness matrix using springs selected based on their positions and directions. Hong and Choi [12] also developed an algorithm for the physical realization of any rank- $r$ isotropic stiffness matrix.

It is easy to understand that the structure of the passive compliance device should be as simple as possible for convenience of engineering implementation. Therefore, the simple springs including the linear springs and torsional springs are preferred to realize a desired stiffness performance. It is worth mentioning that the corresponding wrenches of both the linear spring and the torsional spring are isotropic with zero and infinite pitches, respectively [7].

However, not all of the stiffness matrices can be realized by purely simple springs connected in parallel. The stiffness matrices that can be decomposed into purely isotropic wrenches are 
20-demensional rather than 21-demensitional [4]. In fact, most stiffness matrices would have to be achieved with using screw springs. There are two types of screw springs [13], both of which have nonzero and finite pitches. The structures of the screw springs are shown in Fig.1. When a force is imposed on the connected rigid-body through the screw spring, a coupled torque must be produced synchronously. Hence, the force-deflection characteristics of the screw springs are very complicated. It is unpractical for the physical implementation of a mechanism with several screw springs connected in parallel.

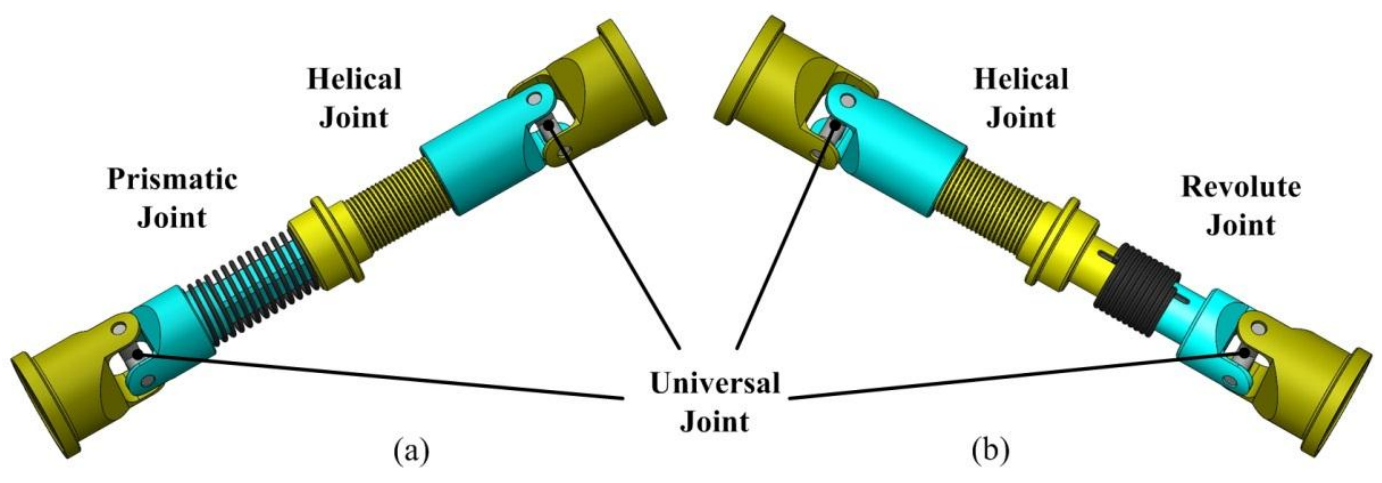

Fig.1 Screw springs. (a) Translational type screw spring (b) Rotational type screw spring.

Roberts [3] has tried to minimize the number of screw springs when realizing a desired stiffness matrix. However, the algorithm cannot completely eliminate the screw springs for most stiffness matrices. As the stiffness matrices are strictly quantitative in mathematics, the described force-deflection characteristics are impossible to be realized precisely for engineering implementation. Inspired by this, the best approximation to stiffness matrix is proposed in this paper. The approximation aims to turn the original complex stiffness matrix into a special approximate matrix so that the desired stiffness performance can be approximately realized by purely simple springs connected in parallel. At this time, the force-deflection characteristics change a little, but the implementation of the mechanism will be easy and practical.

The advantage comes with a price. There must be deviations between the approximate stiffness matrix and the original matrix. Thus an evaluation on the differences is necessary. The existing evaluation induces on the properties of stiffness matrix mainly focus on the eigenvalue [14], determinant [15], trace [16,17], condition number [18], and norm [19] of the matrix. Nevertheless, the physical meaning of these induces is not explicit enough for the entries of stiffness matrix are of heterogeneous units. Meanwhile, many studies concentrating on the structure and mechanical characteristics of the stiffness matrix have also been developed. Typically, Loncaric [20] proposed 
the normal form of stiffness matrix in which the rotational and translational aspects are maximally decoupled. The form is obtained in a special coordinate frame whose origin is termed the center of stiffness [4]. Lipkin et al. [7-9] researched the wrench and twist compliance axes which are very important for they can lend themselves to practical mechanism operations as they define the directions of simple end-effector reactions arising from contact with the surroundings. The centers of the two kinds of compliance axes were proved to be coincident and defined as the center of elasticity [21]. Moreover, El-Khasawneh and Ferreira [22] pointed out that one is usually more interested in the mechanical response of a system in the direction of excitation for many engineering problems, and in consequence, the single-dimensional stiffness defined as the ratio of the magnitude of the wrench to that of the twist is proposed. Portman [23] further put forward the concept of collinear stiffness value (CSV) that embodies the effective stiffness value in one direction. The term collinear means that an initial displacement and resulting force are measured along and about the same axes.

According to the researches above, this paper further presents a physical appreciation on the best approximation. The force-deflection characteristics of the approximate stiffness matrix in different directions are compared with that of the original stiffness matrix. This work can provide guidance for the researchers, to decide whether they can adopt the algorithm to simplify the realization of stiffness requirements.

It is noteworthy that the stiffness matrices mentioned above are all symmetric positive definite/semi-definite (PSD). However, some studies showed that the stiffness matrix relating deflection increment to force increment is not symmetric if a force preload on a mechanism yields significant deflection [24-26]. In this paper, small deflection from a unload equilibrium position is assumed, which means only the symmetric PSD stiffness matrices are taken into account.

The paper is organized as follows: Section 2 presents an overview on the best approximation to stiffness matrix. The motivation and definition as well as the calculation of the approximation are all involved in this section. Next, the physical appreciations of the approximation are provided in Section 3. To illustrate the algorithm intuitively, a numerical example is studied in Section 4. In the end, conclusions are drawn in Section 5.

\section{The best approximation to stiffness matrix}




\subsection{The motivation of the best approximation}

Not all of the $6 \times 6$ symmetric PSD stiffness matrices can be realized by purely simple springs connected in parallel. It has been proved that only when the off-diagonal blocks are traceless, can the symmetric PSD stiffness matrix be decomposed into purely isotropic wrenches [5]. The restrictive condition can be expressed as:

$$
\operatorname{tr}(\mathbf{K} \boldsymbol{\Delta})=0 \quad \boldsymbol{\Delta}=\left[\begin{array}{ll}
\mathbf{0}_{3} & \mathbf{I}_{3} \\
\mathbf{I}_{3} & \mathbf{0}_{3}
\end{array}\right]
$$

where $\operatorname{tr}(*)$ denotes the trace of one matrix. $\mathbf{K}$ is a $6 \times 6$ stiffness matrix while $\Delta$ is an operator. It is easy to verify that $\boldsymbol{\Delta}^{-1}=\boldsymbol{\Delta}^{T}=\boldsymbol{\Delta}$ and $\Delta \boldsymbol{\Delta}=\mathbf{I}_{6}$.

The stiffness matrices satisfying the restriction of formula (1) are isotropic. Obviously, the set consisting of the isotropic stiffness matrices is only a small part of the 21-demensional linear space that contains the whole $6 \times 6$ symmetric stiffness matrices. That is to say, most stiffness matrices would have to be realized with using the screw springs, thus greatly increase the difficulties and costs of physical implementation. For this reason, this paper dedicates to look for a good trade-off between the stiffness requirements and the physical implementation. Consequently, the best approximation which can turn the non-isotropic desired stiffness matrix into an isotropic approximate matrix is put forward.

\subsection{The definition of the best approximation}

The best approximation to stiffness matrix is inspired by the Best Approximation Theorem in Matrix theory [27]. The theorem can be given as follows. Let $U$ be a subspace of $V=\mathbb{R}^{n}, \quad \boldsymbol{\alpha} \in U$ and $\boldsymbol{\beta} \in V$. If

$$
\|\boldsymbol{\beta}-\boldsymbol{\alpha}\|<\|\boldsymbol{\beta}-\boldsymbol{\gamma}\|
$$

holds for any $\boldsymbol{\gamma} \in U$ different from $\boldsymbol{\alpha}$, then $\boldsymbol{\alpha}$ is the best approximation to $\boldsymbol{\beta}$ in the subspace $U$, and can be re-denoted as $\tilde{\boldsymbol{\beta}} .\|\boldsymbol{\beta}-\tilde{\boldsymbol{\beta}}\|$ can be regarded as the error of using $\tilde{\boldsymbol{\beta}}$ in place of $\boldsymbol{\beta}$. From a point of view in geometry, $\tilde{\boldsymbol{\beta}}$ is actually the orthogonal projection of $\boldsymbol{\beta}$ onto $U$, that is $\tilde{\boldsymbol{\beta}}=\operatorname{Proj}_{U} \boldsymbol{\beta}$. Fig.2 shows the best approximation of $\boldsymbol{\beta} \in \mathbb{R}^{3}$ in the two-dimensional subspace. 


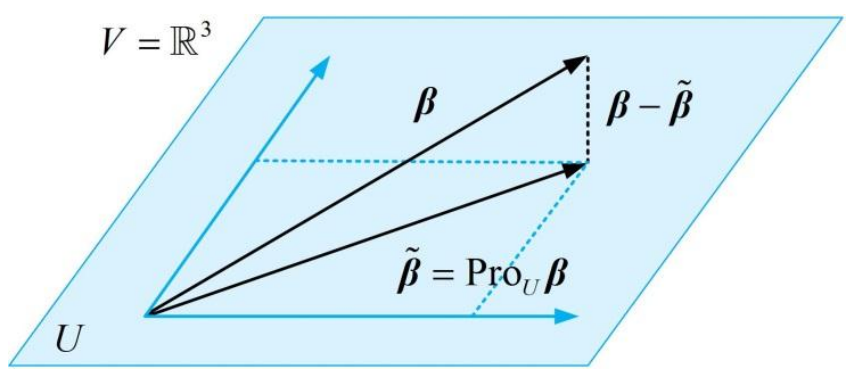

Fig. 2 The best approximation in the three-dimensional linear space.

Based on the best approximation theorem, the best approximation to stiffness matrix is defined. By virtue of this approximation, an isotropic matrix can be derived from the original non-isotropic stiffness matrix.

Definition: Let $V_{\mathbf{K}}=\operatorname{sym}(6), \quad \mathbf{K} \in V_{\mathbf{K}}$ is a $6 \times 6$ symmetric PSD stiffness matrix. If $\tilde{\mathbf{K}} \in U_{\mathbf{K}}$, where $U_{\mathbf{K}} \subseteq V_{\mathbf{K}}:=\left\{\mathbf{K}^{\prime} \in V_{\mathbf{K}} \mid \operatorname{tr}\left(\mathbf{K}^{\prime} \boldsymbol{\Delta}\right)=0\right\}$, then $\tilde{\mathbf{K}}$ is the best approximation to $\mathbf{K}$ in the subspace $U_{\mathbf{K}}$, in the sense that:

$$
\|\mathbf{K}-\tilde{\mathbf{K}}\|<\left\|\mathbf{K}-\mathbf{K}^{\prime}\right\|
$$

for any $\mathbf{K}^{\prime}$ in $U_{\mathbf{K}}$ distinct from $\tilde{\mathbf{K}}$.

Here, $\|\mathbf{K}-\tilde{\mathbf{K}}\|=\sqrt{(\mathbf{K}-\tilde{\mathbf{K}}, \mathbf{K}-\tilde{\mathbf{K}})}$ is the Frobenius norm of the matrix $\mathbf{K}-\tilde{\mathbf{K}} . \quad(*, *)$ denotes the inner product of $\mathbb{R}^{m \times n}$, which is given by $(\mathbf{M}, \mathbf{N})=\operatorname{tr}\left(\mathbf{M}^{T} \mathbf{N}\right) .\|\mathbf{K}-\tilde{\mathbf{K}}\|$ can be seen as a measure on how much the matrix $\mathbf{K}-\tilde{\mathbf{K}}$ differs from zero [19]. Therefore, $\|\mathbf{K}-\tilde{\mathbf{K}}\|$ embodies the difference between the original stiffness matrix and the approximate matrix. Important to note is that $V_{\mathbf{K}}$ is 21-demensional while $U_{\mathbf{K}}$ is 20-demensional. The best approximation to stiffness matrix has the following characteristics:

Proposition 1: $\tilde{\mathbf{K}}$ is the best approximation to $\mathbf{K} \Leftrightarrow \mathbf{K}-\tilde{\mathbf{K}} \in U_{K}^{\perp}$.

Proof: According to the definition of best approximation to stiffness matrix, the formula:

$$
\begin{aligned}
\left\|\mathbf{K}-\mathbf{K}^{\prime}\right\|^{2} & =\left\|(\mathbf{K}-\tilde{\mathbf{K}})+\left(\tilde{\mathbf{K}}-\mathbf{K}^{\prime}\right)\right\|^{2} \\
& =\|\mathbf{K}-\tilde{\mathbf{K}}\|^{2}+\left\|\tilde{\mathbf{K}}-\mathbf{K}^{\prime}\right\|^{2}+2\left(\mathbf{K}-\tilde{\mathbf{K}}, \tilde{\mathbf{K}}-\mathbf{K}^{\prime}\right) \\
& >\|\mathbf{K}-\tilde{\mathbf{K}}\|^{2}
\end{aligned}
$$

holds for any $\mathbf{K}^{\prime}$ distinct from $\tilde{\mathbf{K}}$. Therefore, $\left\|\tilde{\mathbf{K}}-\mathbf{K}^{\prime}\right\|^{2}+2\left(\mathbf{K}-\tilde{\mathbf{K}}, \tilde{\mathbf{K}}-\mathbf{K}^{\prime}\right)$ must be positive 
so that $\left(\mathbf{K}-\tilde{\mathbf{K}}, \tilde{\mathbf{K}}-\mathbf{K}^{\prime}\right)$ should equal to zero. Since $\tilde{\mathbf{K}}-\mathbf{K}^{\prime} \in U_{\mathbf{K}}$, then $\mathbf{K}-\tilde{\mathbf{K}} \in U_{\mathbf{K}}^{\perp}$.

Proposition 2: For an arbitrary non-isotropic stiffness matrix $\mathbf{K} \in V_{\mathbf{K}}$, the best-approximate isotropic matrix $\tilde{\mathbf{K}} \in U_{\mathbf{K}}$ is unique.

Proof: Assume $\hat{\mathbf{K}} \in U_{\mathbf{K}}$ is another best-approximate isotropic matrix of $\mathbf{K}$. Then,

$$
\|\mathbf{K}-\tilde{\mathbf{K}}\|=\|\mathbf{K}-\widehat{\mathbf{K}}\|
$$

To expand the formula (5), then the equation $(\tilde{\mathbf{K}}, \tilde{\mathbf{K}})-(\widehat{\mathbf{K}}, \widehat{\mathbf{K}})=2(\mathbf{K}, \tilde{\mathbf{K}}-\widehat{\mathbf{K}})$ can be derived. Replace the $\tilde{\mathbf{K}}$ by $\left(\widehat{\mathbf{K}}+\mathbf{K}_{0}\right)$, where $\mathbf{K}_{0} \in U_{\mathbf{K}}$. Note that $\mathbf{K}-\widehat{\mathbf{K}}$ belongs to the $U_{\mathbf{K}}^{\perp}$ in terms of the proposition 1. Finally, we can obtain that:

$$
\left(\mathbf{K}_{0}, \mathbf{K}_{0}\right)=2\left(\mathbf{K}_{0}, \mathbf{K}-\widehat{\mathbf{K}}\right)=0
$$

That is to say, $\mathbf{K}_{0}=\mathbf{0}_{6}$ and $\widehat{\mathbf{K}}=\tilde{\mathbf{K}}$. The best approximate to stiffness matrix is unique.

\subsection{The calculation of the best approximation}

The best approximation in $\mathbb{R}^{n}$ is actually the orthogonal projection of a vector onto one subspace. Similarly, the best approximation to stiffness matrix can be calculated.

Proposition 3: Let $\mathbf{K} \in V_{\mathbf{K}}$, then the best-approximate isotropic matrix $\tilde{\mathbf{K}}$ is the orthogonal projection of $\mathbf{K}$ onto $U_{\mathbf{K}}$, that is:

$$
\tilde{\mathbf{K}}=\operatorname{Pro}_{U_{\mathbf{K}}} \mathbf{K}=\sum_{i=1}^{20}\left(\mathbf{K}, \boldsymbol{\Omega}_{i}\right) \boldsymbol{\Omega}_{i}
$$

where $\left\{\begin{array}{llll}\boldsymbol{\Omega}_{1} & \cdots & \boldsymbol{\Omega}_{20}\end{array}\right\}$ is an orthonormal set of $U_{\mathbf{K}}$ satisfying:

$$
\left(\boldsymbol{\Omega}_{i}, \boldsymbol{\Omega}_{j}\right)=\left\{\begin{array}{ll}
1, & i=j \\
0, & i \neq j
\end{array} \quad \boldsymbol{\Omega}_{i}, \boldsymbol{\Omega}_{j} \in U_{\mathbf{K}}\right.
$$

It is easy to verify that $(\mathbf{K}-\tilde{\mathbf{K}}, \tilde{\mathbf{K}})=0$ at this time, which means $(\mathbf{K}-\tilde{\mathbf{K}}) \in U_{\mathbf{K}}^{\perp}$. According to the proposition 1, the $\tilde{\mathbf{K}}$ in formula (7) is the best approximation of $\mathbf{K}$.

The following presents a set of orthonormal bases of $U_{\mathbf{K}}$. In terms of the positions of the nonzero entries, the orthonormal set can be divided into three subgroups denoted as $\left\{\boldsymbol{\Omega}_{i i}\right\},\left\{\boldsymbol{\Omega}_{i j}\right\}$ and $\left\{\boldsymbol{\Omega}_{B}\right\}$. For the matrices in the first subgroup, the entries of the matrix are all zero except the 
$(i, i)$ entry equaling to one. The size of this subgroup is six. For the bases in the second subgroup, the $(i, j)$ and $(j, i)$ entries are $\sqrt{2} / 2$ while the other entries are all zero. Here, $i \neq j$ and $(i, j)$ does not refer to the diagonal entries of the off-diagonal blocks of matrix. The number of this group of bases is twelve. At last, there are still two bases whose off-diagonal blocks are $\operatorname{diag}\left(\begin{array}{lll}1 / 2 & 1 / 2 & 0\end{array}\right)$ and $\operatorname{diag}(\sqrt{3} / 6 \quad \sqrt{3} / 6 \quad-\sqrt{3} / 3)$, respectively. The orthonormal bases are summarized in Table.1.

Table.1 An orthonormal set of $U_{\mathbf{K}}$.

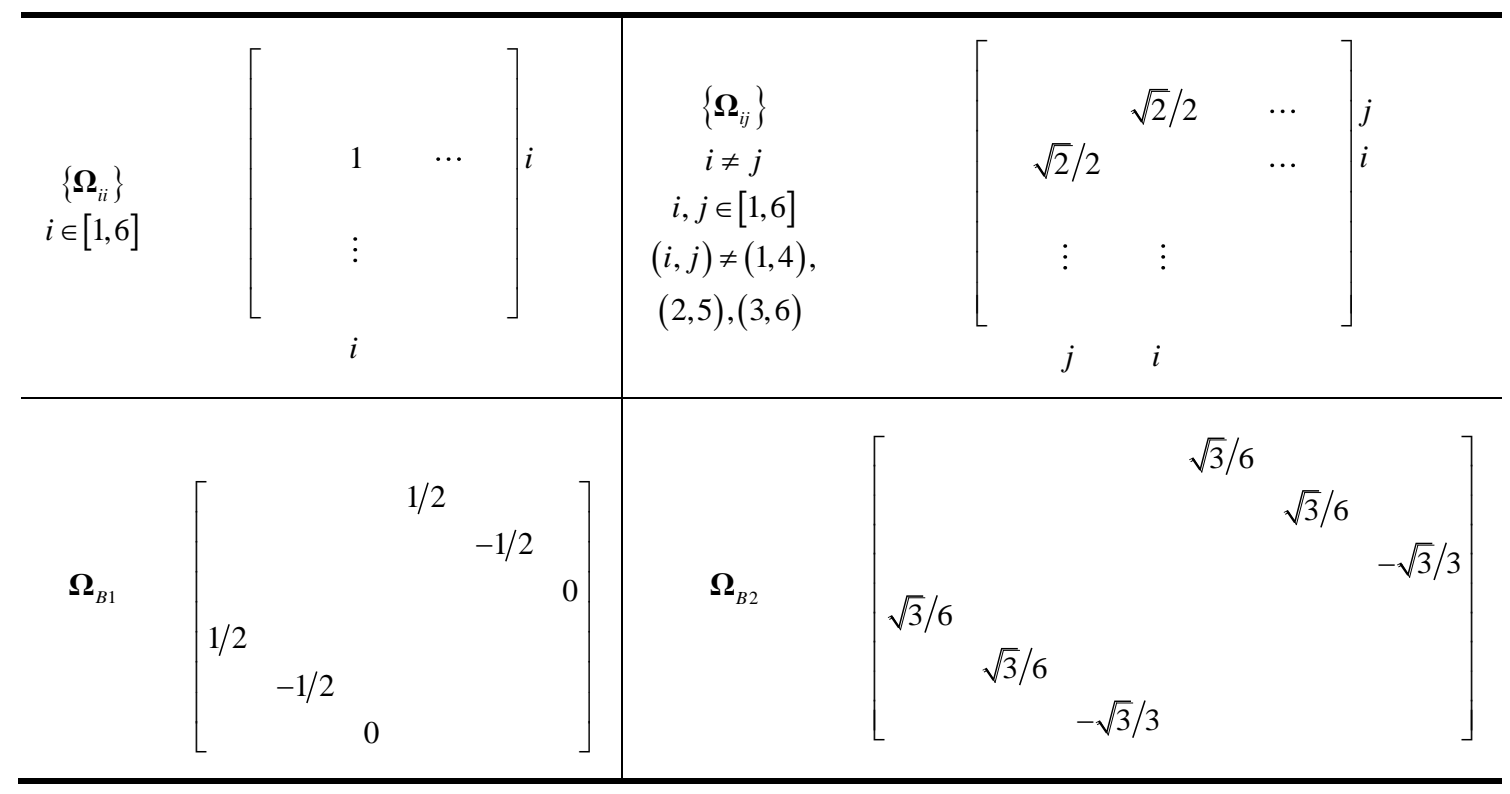

The formula (7) is not simple enough. Next, a more concise formula is provided.

Proposition 4: Let $a, b$ and $c$ be the diagonal entries of the off-diagonal blocks of $\mathbf{K}$, then the corresponding entries of $\tilde{\mathbf{K}}$ are changed to:

$$
\left\{\begin{array}{l}
\tilde{a}=a-\operatorname{tr}(\mathbf{K} \mathbf{\Delta}) / 6 \\
\tilde{b}=b-\operatorname{tr}(\mathbf{K} \mathbf{\Delta}) / 6 \\
\tilde{c}=c-\operatorname{tr}(\mathbf{K} \mathbf{\Delta}) / 6
\end{array}\right.
$$

while the rest entries all remain unchanged. That is to say:

$$
\tilde{\mathbf{K}}=\mathbf{K}-\mu \Delta
$$

where $\mu=\operatorname{tr}(\mathbf{K \Delta}) / 6=(a+b+c) / 3$.

Proof: The Proposition 2 has proved that the best approximation to any symmetric stiffness matrix is unique. In other words, although the orthonormal sets of $U_{\mathbf{K}}$ are varied, the resultant ap- 
proximate matrix should always be the same, regardless of which set you choose. Accordingly, the orthonormal bases given in Table.1 are used. The following results can be derived:

$$
\begin{aligned}
& \left(\mathbf{K}, \boldsymbol{\Omega}_{i i}\right)=\operatorname{tr}\left(\mathbf{K}^{T} \boldsymbol{\Omega}_{i i}\right)=K_{i i} \quad i \in[1,6] \\
& \left(\mathbf{K}, \boldsymbol{\Omega}_{i j}\right)=\operatorname{tr}\left(\mathbf{K}^{T} \boldsymbol{\Omega}_{i j}\right)=\sqrt{2} K_{i j} \quad i \neq j, i, j \in[1,6] \quad(i, j) \neq(4,1),(5,2),(6,3) \\
& \left(\mathbf{K}, \boldsymbol{\Omega}_{B 1}\right)=\operatorname{tr}\left(\mathbf{K}^{T} \boldsymbol{\Omega}_{B 1}\right)=K_{41}-K_{52} \\
& \left(\mathbf{K}, \boldsymbol{\Omega}_{B 2}\right)=\operatorname{tr}\left(\mathbf{K}^{T} \mathbf{\Omega}_{B 2}\right)=\frac{\sqrt{3}}{3} K_{41}+\frac{\sqrt{3}}{3} K_{52}-\frac{2 \sqrt{3}}{3} K_{63}
\end{aligned}
$$

where $K_{i j}$ is the $(i, j)$ entry of the stiffness matrix $\mathbf{K}$. To substitute the results into formula (7), it is easy to verify that $\tilde{\mathbf{K}}=\mathbf{K}-\mu \boldsymbol{\Delta}$.

Based on the analyses above, we can finally recognize the minimal 'distance' between the $\mathbf{K} \in V_{\mathbf{K}}$ and $\mathbf{K}^{\prime} \in U_{\mathbf{K}}$. The 'distance' provides a judgement on how much the differences between the original stiffness matrix and the approximate matrix are in mathematics. That is:

$$
D_{\min }=\|\mathbf{K}-\tilde{\mathbf{K}}\|=\|\mu \Delta\|=\frac{\sqrt{6}}{3}(a+b+c)
$$

Although $V_{\mathbf{K}}=\operatorname{sym}(6)$ is a 21-dimensional linear space, the set comprised by the whole $6 \times 6$ symmetric PSD stiffness matrices is in fact a cone in $V_{\mathbf{K}}$. However, the definition of best approximation to stiffness matrix is in the linear space, which means the resultant approximate matrix $\tilde{\mathbf{K}}$ is not necessarily positive definite/semi-definite. As a result, $\tilde{\mathbf{K}}$ cannot be realized by general springs connected in parallel unless $\tilde{\mathbf{K}}$ is a PSD matrix. Fig.3 presents an interpretation on the limitation of best approximation to stiffness matrix.

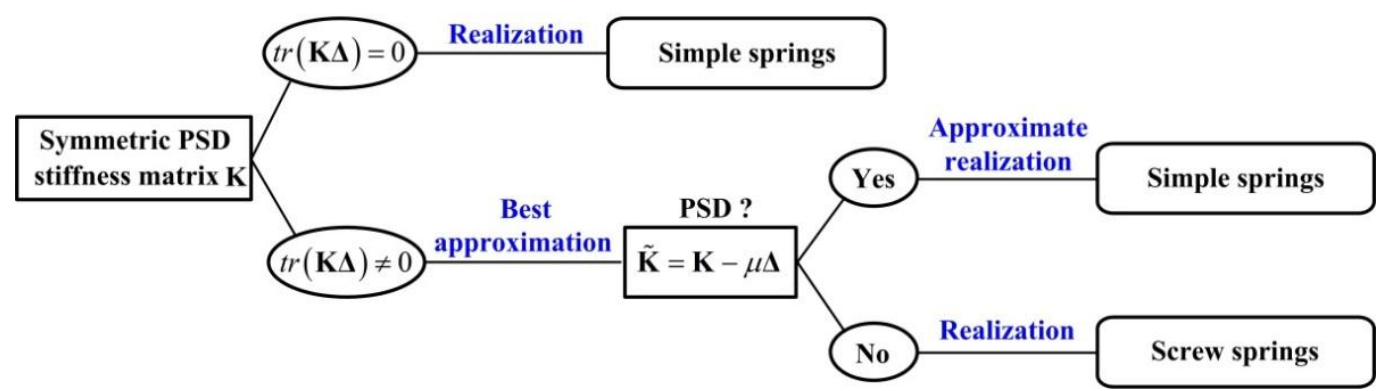

Fig.3 The limitation of the best approximation to stiffness matrix. For an arbitrary symmetric PSD stiffness matrix $\mathbf{K}$, it can be realized by purely simple springs connected in parallel when $\mathbf{K}$ is isotropic; otherwise, if the best approximation $\tilde{\mathbf{K}}$ is PSD, $\mathbf{K}$ can be approximately realized by purely simple springs, else $\mathbf{K}$ can only be realized by using screw springs.

\section{The physical appreciations of the best approximation}

\subsection{The best approximation versus the coordinate transformation}


The stiffness matrix depends on the choice of reference coordinate frame. Especially for the general stiffness decomposition algorithms such as the eigenvalue decomposition [28] and Cholesky decomposition [5], the change of coordinate frame may bring different spring wrenches. Will the coordinate transformation lead to a different best approximation to the desired stiffness matrix?

The coordinate transformation of spatial stiffness matrix preserves in the form of congruence transformation [20]. Let the twist deflection of a rigid-body suspended by several elastic units be represented in Plücker's axis coordinate while the wrench exerting on it be represented in Plücker's ray coordinate. Then the coordinate transformation of spatial stiffness matrix can be written as:

$$
\mathbf{K}^{P}=\operatorname{Ad}_{\mathbf{g}_{O P}}^{T} \mathbf{K}^{O} \operatorname{Ad}_{\mathbf{g}_{O P}} \quad \operatorname{Ad}_{\mathbf{g}_{O P}}=\left[\begin{array}{cc}
\mathbf{R} & \hat{\mathbf{r}} \mathbf{R} \\
\mathbf{0}_{3} & \mathbf{R}
\end{array}\right] \quad \mathbf{g}_{O P}=\left[\begin{array}{cc}
\mathbf{R} & \boldsymbol{r} \\
\boldsymbol{0}_{1 \times 3} & 1
\end{array}\right]
$$

where $\mathbf{K}^{P}$ and $\mathbf{K}^{O}$ represent the stiffness matrices expressed in frame $\{P\}$ and frame $\{O\}$, respectively. $\mathrm{Ad}_{\mathbf{g}_{O P}}$ is the adjoint operator associating with $\mathbf{g}_{O P} \in S E(3)$ which is the homogeneous transformation matrix of frame $\{P\}$ with respect to frame $\{O\}$ [29]. For brevity, the $\operatorname{Ad}_{\mathbf{g}_{o p}}$ is abbreviated as $\mathrm{Ad}_{\mathrm{g}}$ in the following analyses. $\hat{\mathbf{r}} \in \operatorname{so}(3)$ is the skew-symmetric matrix associating with the position vector $\boldsymbol{r}$ of point $P$ relative to point $O$. After verification, the equation $\operatorname{Ad}_{\mathrm{g}}{ }^{T} \Delta \mathrm{Ad}_{\mathrm{g}}=\operatorname{Ad}_{\mathrm{g}} \Delta \operatorname{Ad}_{\mathrm{g}}{ }^{T}=\boldsymbol{\Delta}$ is true. Hence, the best approximation of $\mathbf{K}^{P}$ can be calculated on the basis of formula (10) as:

$$
\tilde{\mathbf{K}}^{P}=\mathbf{K}^{P}-\frac{\operatorname{tr}\left(\mathbf{K}^{P} \boldsymbol{\Delta}\right)}{6} \boldsymbol{\Delta}=\operatorname{Ad}_{\mathbf{g}}{ }^{T} \mathbf{K}^{o} \operatorname{Ad}_{\mathrm{g}}-\frac{\operatorname{tr}\left(\operatorname{Ad}_{\mathrm{g}}{ }^{T} \mathbf{K}^{O} \operatorname{Ad}_{\mathbf{g}} \boldsymbol{\Delta}\right)}{6} \operatorname{Ad}_{\mathbf{g}}{ }^{T} \Delta \operatorname{Ad}_{\mathbf{g}}
$$

Moreover, it can be derived that $\operatorname{tr}\left(\operatorname{Ad}_{\mathrm{g}}{ }^{T} \mathbf{K}^{o} \operatorname{Ad}_{\mathrm{g}} \boldsymbol{\Delta}\right)=\operatorname{tr}\left(\mathbf{K}^{o} \operatorname{Ad}_{\mathrm{g}} \Delta \mathrm{Ad}_{\mathrm{g}}{ }^{T}\right)=\operatorname{tr}\left(\mathbf{K}^{o} \boldsymbol{\Delta}\right)$, therefore:

$$
\tilde{\mathbf{K}}^{P}=\operatorname{Ad}_{\mathbf{g}}{ }^{T}\left(\mathbf{K}^{o}-\frac{\operatorname{tr}\left(\mathbf{K}^{o} \boldsymbol{\Delta}\right)}{6} \boldsymbol{\Delta}\right) \operatorname{Ad}_{\mathbf{g}}=\operatorname{Ad}_{\mathbf{g}}{ }^{T} \tilde{\mathbf{K}}^{o} \operatorname{Ad}_{\mathbf{g}}
$$

To compare the result with formula (13), it is clear that the best approximation to stiffness matrix keeps the same form of congruence transformation. That is to say, the best approximation is independent with the coordinate transformation. Not only in mathematics, but in physics, the best approximation to stiffness matrix is unique. 


\subsection{The norm form and center of stiffness}

The $6 \times 6$ symmetric PSD stiffness matrix $\mathbf{K}$ can be partitioned into $3 \times 3$ blocks in the form:

$$
\mathbf{K}=\left[\begin{array}{cc}
\mathbf{A} & \mathbf{B} \\
\mathbf{B}^{T} & \mathbf{D}
\end{array}\right]
$$

where the upper left block-matrix $\mathbf{A}$ and the lower right block-matrix $\mathbf{D}$, respectively, denote the translational and rotational aspects. Meanwhile, B is the coupling aspect. Similarly, the best-approximate isotropic stiffness matrix $\tilde{\mathbf{K}}$ can be represented as:

$$
\tilde{\mathbf{K}}=\left[\begin{array}{cc}
\mathbf{A} & \tilde{\mathbf{B}} \\
\tilde{\mathbf{B}}^{T} & \mathbf{D}
\end{array}\right]
$$

where $\tilde{\mathbf{B}}=\mathbf{B}-\mu \mathbf{I}_{3}$. Obviously, the best approximation does not change the translational and the rotational aspects except the coupling aspect of the original stiffness matrix.

In order to maximally decouple the rotational and translational aspects, the norm form of stiffness matrix was proposed in which the off-diagonal blocks $\mathbf{B}$ are diagonalized [20]. The form can be obtained by choosing a particular reference coordinate frame $\{\underline{O}\}$, and the origin of the frame is termed the center of stiffness. It is easy to derive the stiffness matrix expressed in frame $\{\underline{Q}\}$. That is:

$$
\underline{\mathbf{K}}=\left[\begin{array}{cc}
\underline{\mathbf{A}} & \underline{\mathbf{B}} \\
\underline{\mathbf{B}}^{T} & \underline{\mathbf{D}}
\end{array}\right]=\left[\begin{array}{cc}
\mathbf{R}^{T} \mathbf{A} \mathbf{R} & \mathbf{R}^{T} \mathbf{A} \hat{\mathbf{r}} \mathbf{R}+\mathbf{R}^{T} \mathbf{B R} \\
\mathbf{R}^{T} \hat{\mathbf{r}}^{T} \mathbf{A} \mathbf{R}+\mathbf{R}^{T} \mathbf{B}^{T} \mathbf{R} & \mathbf{R}^{T} \hat{\mathbf{r}}^{T} \mathbf{A} \hat{\mathbf{r}} \mathbf{R}+\mathbf{R}^{T} \mathbf{B}^{T} \hat{\mathbf{r}} \mathbf{R}+\mathbf{R}^{T} \hat{\mathbf{r}}^{T} \mathbf{B R}+\mathbf{R}^{T} \mathbf{D R}
\end{array}\right]
$$

where $\underline{\mathbf{K}}$ denotes the norm form of the original stiffness matrix, and $\underline{\mathbf{B}}$ is a diagonal matrix.

Transform $\tilde{\mathbf{K}}$ into this particular coordinate frame. The representation of $\tilde{\mathbf{K}}$ in frame $\{\underline{O}\}$ can be obtained by replacing $\mathbf{B}$ in formula (18) by $\tilde{\mathbf{B}}$. It can be verified that $\underline{\tilde{\mathbf{K}}}=\underline{\mathbf{K}}-\mu \boldsymbol{\Delta}$ for $\mathbf{R}^{T} \mathbf{R}=\mathbf{I}_{3}$. Clearly, the coupling aspect $\underline{\tilde{\mathbf{B}}}=\underline{\mathbf{B}}-\mu \mathbf{I}_{3}$ is still a diagonal matrix, which means the best approximation of $\mathbf{K}$ is also in the norm form in frame $\{\underline{Q}\}$. In other words, the best approximation does not vary the center of stiffness of the original stiffness matrix. Interesting to note is that $\underline{\mu}=\operatorname{tr}(\underline{\mathbf{K}} \mathbf{\Lambda}) / 6=\mu$. Therefore, $\underline{\tilde{\mathbf{K}}}$ is also the best approximation of $\underline{\mathbf{K}}$. To illustrate the relationships between the four special stiffness matrices, the Fig.4 is drawn, in which the hollow arrow represents the process of best approximation and the solid arrow represents the process of 
coordinate transformation (as well as the process turning a stiffness matrix into its norm form).

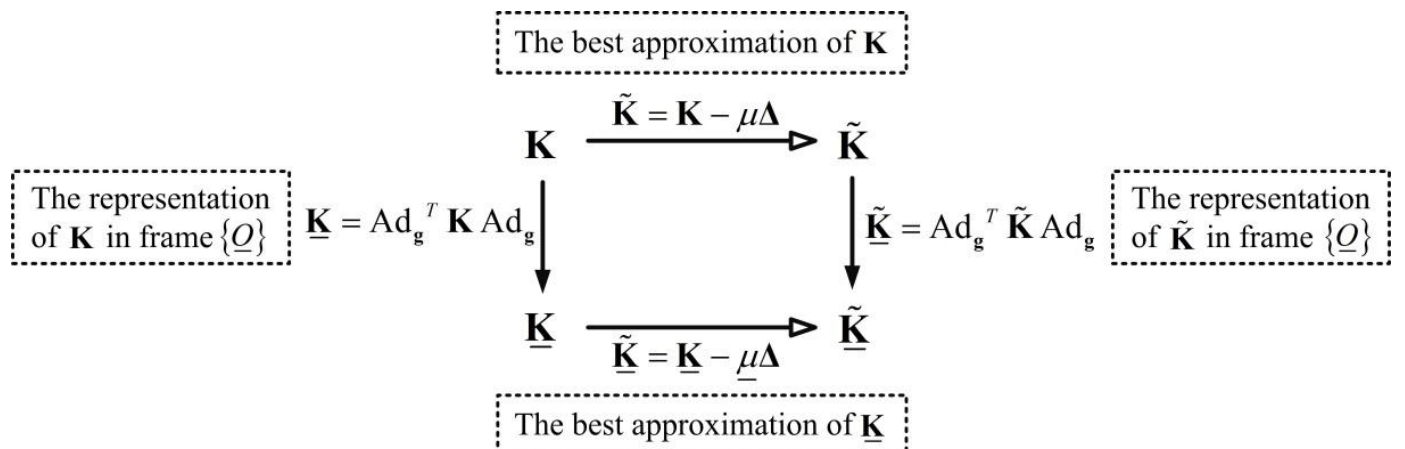

Fig.4 Relationships between four special stiffness matrices.

\subsection{The wrench/twist compliance axes and center of elasticity}

The wrench and twist compliance axis can be determined by eigenwrench and eigentwist [9]. The centers of the wrench compliance axes and twist compliance axes are coincident, and this unique point is termed the center of elasticity [21]. Let $\mathbf{C}=\mathbf{K}^{-1}$ be the compliance matrix. For a nonsingular elastic system, the matrix can be written as:

$$
\mathbf{C}=\left[\begin{array}{cc}
\mathbf{A}^{-1}+\mathbf{A}^{-1} \mathbf{B} \mathbf{H} \mathbf{B}^{T} \mathbf{A}^{-1} & -\mathbf{A}^{-1} \mathbf{B H} \\
-\mathbf{H B}^{T} \mathbf{A}^{-1} & \mathbf{H}
\end{array}\right]
$$

where $\mathbf{H}=\left(\mathbf{D}-\mathbf{B}^{T} \mathbf{A}^{-1} \mathbf{B}\right)^{-1}$. Then the eigenwrench and eigentwist can be obtained, as follows:

$$
\text { eigenwrench : }\left[\begin{array}{c}
\boldsymbol{f}_{i} \\
\frac{1}{\lambda_{i}} \mathbf{B}^{T} \boldsymbol{f}_{i}
\end{array}\right] \quad \text { eigentwist : }\left[\begin{array}{c}
-\mathbf{A}^{-1} \mathbf{B} \boldsymbol{\varphi}_{i} \\
\boldsymbol{\varphi}_{i}
\end{array}\right]
$$

where $\boldsymbol{f}_{i} \in \mathbb{R}^{3}$ and $\lambda_{i}$ are the eigenvector and eigenvalue of $\mathbf{A}$, and $\boldsymbol{\varphi}_{i} \in \mathbb{R}^{3}$ and $\eta_{i}$ are the eigenvector and eigenvalue of $\mathbf{H}$. On these bases, the center of elasticity can be identified according to the geometries of the eigenwrenches or eigentwists.

Obviously, $f_{i}$ and $\varphi_{i}$ specify the directions of the wrench and twist compliance axis, respectively. To determine the position of the wrench compliance axis, the eigenwrench is rewritten as:

$$
\left[\begin{array}{c}
\boldsymbol{f}_{i} \\
\frac{1}{\lambda_{i}} \mathbf{B}^{T} \boldsymbol{f}_{i}
\end{array}\right]=\left[\begin{array}{c}
\boldsymbol{f}_{i} \\
\boldsymbol{r}_{i}^{w} \times \boldsymbol{f}_{i}+h_{i}^{w} \boldsymbol{f}_{i}
\end{array}\right]
$$

where $h_{i}^{w}$ is the pitch, and $r_{i}^{w}$ is the coordinates of one point on the wrench compliance axis. The coordinates can be obtained by:

$$
\boldsymbol{r}_{i}^{w}=\boldsymbol{f}_{i} \times\left(\boldsymbol{r}_{i}^{w} \times \boldsymbol{f}_{i}\right)=\boldsymbol{f}_{i} \times\left(\frac{1}{\lambda_{i}} \mathbf{B}^{T} \boldsymbol{f}_{i}-h_{i}^{w} \boldsymbol{f}_{i}\right)=\frac{1}{\lambda_{i}} \hat{\mathbf{f}}_{i} \mathbf{B}^{T} \boldsymbol{f}_{i}
$$


Analogously, the position of the twist compliance axis can be calculated by:

$$
\boldsymbol{r}_{i}^{t}=\boldsymbol{\varphi}_{i} \times\left(\boldsymbol{r}_{i}^{t} \times \boldsymbol{\varphi}_{i}\right)=-\hat{\boldsymbol{\varphi}}_{i} \mathbf{A}^{-1} \mathbf{B} \boldsymbol{\varphi}_{i}
$$

As the best approximation does not change the translational aspect of stiffness matrix, the directions of the wrench compliance axes are invariant. Moreover, it can be verified that:

$$
\tilde{\boldsymbol{r}}_{i}^{w}=\frac{1}{\lambda_{i}} \hat{\hat{\mathbf{f}}_{i}} \tilde{\mathbf{B}}^{T} \boldsymbol{f}_{i}=\frac{1}{\lambda_{i}} \hat{\mathbf{f}}_{i} \mathbf{B}^{T} \boldsymbol{f}_{i}-\frac{\mu}{\lambda_{i}} \boldsymbol{f}_{i} \times \boldsymbol{f}_{i}=\frac{1}{\lambda_{i}} \hat{\mathbf{f}}_{i} \mathbf{B}^{T} \boldsymbol{f}_{i}=\boldsymbol{r}_{i}^{w}
$$

which means the positions of the wrench compliance axes are also changeless after the best approximation. On the other hand, since the matrix $\tilde{\mathbf{H}}$ is different from $\mathbf{H}$, then the twist compliance axes would be changed because of the approximation. Nevertheless, regardless of the changes happened on the twist compliance axes, the center of elasticity is invariable for the geometries of the wrench compliance axes are changeless.

\subsection{The translational and rotational stiffness value}

Although the $6 \times 6$ stiffness matrix can provide an overall reveal on the force-deflection characteristics, the desired stiffness properties are normally focused on some special directions for many engineering applications. For example, in the polishing and grinding operations, the stiffness is required usually in the direction perpendicular to the contact surface with the workpiece. Since the single-dimensional stiffness [22] was defined as the ratio of the wrench to twist rather than the force to displacement or the torque to angle, the dimension of the concept is not very clear. By contrast, the translational stiffness value (TSV) and rotational stiffness value (RSV) [23] are more meaningful for they can help to quantify the force-translational deflection and the torque-rotational deflection characteristics in one special direction from a sophisticated stiffness matrix.

The TSV is defined as the ratio of the magnitude of the response force coinciding with the direction of deflection to that of the unit translational deflection. The ratio can be calculated as:

$$
k_{t r}=\boldsymbol{X}_{t r}^{T} \mathbf{K} \boldsymbol{X}_{t r}=\boldsymbol{\delta}^{T} \mathbf{A} \boldsymbol{\delta}
$$

where $\boldsymbol{X}_{t r}=\left[\begin{array}{ll}\boldsymbol{\delta}^{T} & \boldsymbol{O}_{3 \times 1}^{T}\end{array}\right]^{T}$ represents an arbitrary unit translational deflection acting on the suspended rigid-body. Find that $\boldsymbol{X}_{t r}^{T} \mathbf{K} \boldsymbol{X}_{t r}$ also depicts the deformation potential energy resulted from the translational deflection. As the best approximation does not change the translational aspect of stiffness matrix, the TSV is invariant. The result also indicates that the deformation poten- 
tial energy is changeless after the approximation when an arbitrary translational deflection is exerted on the suspended rigid-body.

On the other hand, the RSV is defined as the ratio of the magnitude of the coaxial response resultant torque to that of the rotational deflection. Let $\quad \boldsymbol{X}_{r o t}=\left[\begin{array}{ll}(\boldsymbol{r} \times \boldsymbol{\varphi})^{T} & \boldsymbol{\varphi}^{T}\end{array}\right]^{T}$ denotes an arbitrary unit rotational deflection around an axis passing through one point $P$, where $r \in \mathbb{R}^{3}$ is the vector from the origin $O$ to the point $P$. Then the RSV can be ultimately obtained as:

$$
k_{r o t}=\boldsymbol{X}_{r o t}^{T} \mathbf{K} \boldsymbol{X}_{r o t}=\boldsymbol{\varphi}^{T}\left(\hat{\mathbf{r}}^{T} \mathbf{A} \hat{\mathbf{r}}+\hat{\mathbf{r}}^{T} \mathbf{B}+\mathbf{B}^{T} \hat{\mathbf{r}}+\mathbf{D}\right) \boldsymbol{\varphi}
$$

Note that $\boldsymbol{X}_{r o t}^{T} \mathbf{K} \boldsymbol{X}_{r o t}$ also embodies the deformation potential energy caused by the rotational deflection. As the coupling aspect changes to $\tilde{\mathbf{B}}=\mathbf{B}-\mu \mathbf{I}_{3}$ and the other aspects of stiffness matrix are invariant after the best approximation, it is not hard to verify that $\tilde{k}_{r o t}$ equals to $k_{\text {rot }}$ for $\hat{\mathbf{r}}^{T}=-\hat{\mathbf{r}}$. That is to say, the best approximation does not change the RSV. The result also indicates that the deformation potential energy arising from an arbitrary rotational deflection is the same after the best approximation.

\section{Numerical Example}

In this section, one numerical example is studied to demonstrate the best-approximate realization of the non-isotropic stiffness requirements by purely simple springs connected in parallel. The desired stiffness matrix is designed as follows:

$$
\mathbf{K}=\left[\begin{array}{cccccc}
69 & -22 & 15 & 6 & 12 & 11 \\
-22 & 77 & 7 & 29 & 14 & 25 \\
15 & 7 & 44 & 28 & 1 & -14 \\
6 & 29 & 28 & 103 & 17 & -13 \\
12 & 14 & 1 & 17 & 73 & 17 \\
11 & 25 & -14 & -13 & 17 & 105
\end{array}\right]
$$

Since $\operatorname{tr}(\mathbf{K} \mathbf{\Delta})=12$ for this case, the stiffness matrix is non-isotropic and cannot be realized by purely simple springs connected in parallel. Moreover, it has been proved that at least $q$ screw springs are required to realize this stiffness matrix, where $q$ is the minimal positive integer satisfying [3]:

$$
\lambda_{1}+\cdots+\lambda_{q} \leq \operatorname{tr}(\mathbf{K} \mathbf{\Delta}) \leq \lambda_{r+1-q}+\cdots+\lambda_{r}
$$


Here, $\lambda_{1} \leq \cdots \leq \lambda_{r}$ represent the ordered nonzero eigenvalues of $\mathbf{K \Delta}$, i.e., $-102.0007,-68.0050$, $-38.0990,33.6762,65.9771$, and 120.4516. According to the formula (28), at least one screw spring is needed to realize the desired stiffness matrix. The best-approximate matrix can be easily derived as:

$$
\tilde{\mathbf{K}}=\left[\begin{array}{cccccc}
69 & -22 & 15 & 4 & 12 & 11 \\
-22 & 77 & 7 & 29 & 12 & 25 \\
15 & 7 & 44 & 28 & 1 & -16 \\
4 & 29 & 28 & 103 & 17 & -13 \\
12 & 12 & 1 & 17 & 73 & 17 \\
11 & 25 & -16 & -13 & 17 & 105
\end{array}\right]
$$

Obviously, $\tilde{\mathbf{K}}$ is positive definite, and the trace of the off-diagonal blocks is zero. Therefore, $\tilde{\mathbf{K}}$ is an isotropic stiffness matrix.

\subsection{The implementation of the stiffness requirements}

In order to illustrate the advantages of the best approximation, the implementations of the original stiffness matrix $\mathbf{K}$ and the best-approximate isotropic stiffness matrix $\tilde{\mathbf{K}}$ are all provided. More specifically, three common stiffness decomposition algorithms, including the eigenscrew decomposition [6], the compliance axes decomposition [8] and the minimal decomposition [3], are adopted to realize the $\mathbf{K}$. Meanwhile, the decomposition algorithm proposed in [10] is used to realize the $\tilde{\mathbf{K}}$.

The results show that, to meet the stiffness matrix precisely, six screw springs are needed for the eigenscrew decomposition, while three screw springs and three torsional springs are required for the compliance axes decomposition. Even for the minimal decomposition, one screw spring is still required. By contrast, combining with the best approximation, six linear springs connected in parallel can approximately achieve the desired stiffness requirements. Obviously, the best approximation can significantly simplify the physical implementation when meeting the non-isotropic stiffness requirements. The analysis results are summarized in Table.2. The physical realizations of the four kinds of algorithms are presented in Fig.5, in which the red vectors represent the directions of the screw springs and the blue vectors denote the directions of the simple springs. Moreover, a sketch of a passive compliance apparatus is shown in Fig.6. The apparatus is designed by the best approximation, and the force-deflection characteristics of the end-effector are satisfied with the 
best-approximate isotropic stiffness matrix $\tilde{\mathbf{K}}$ in frame $\{O\}$. It is noted that the spring wrench specifies the position, direction and type of the passive spring, nevertheless, the spring can be arbitrarily extended or translated along the wrench as long as the stiffness constant is unchanged.
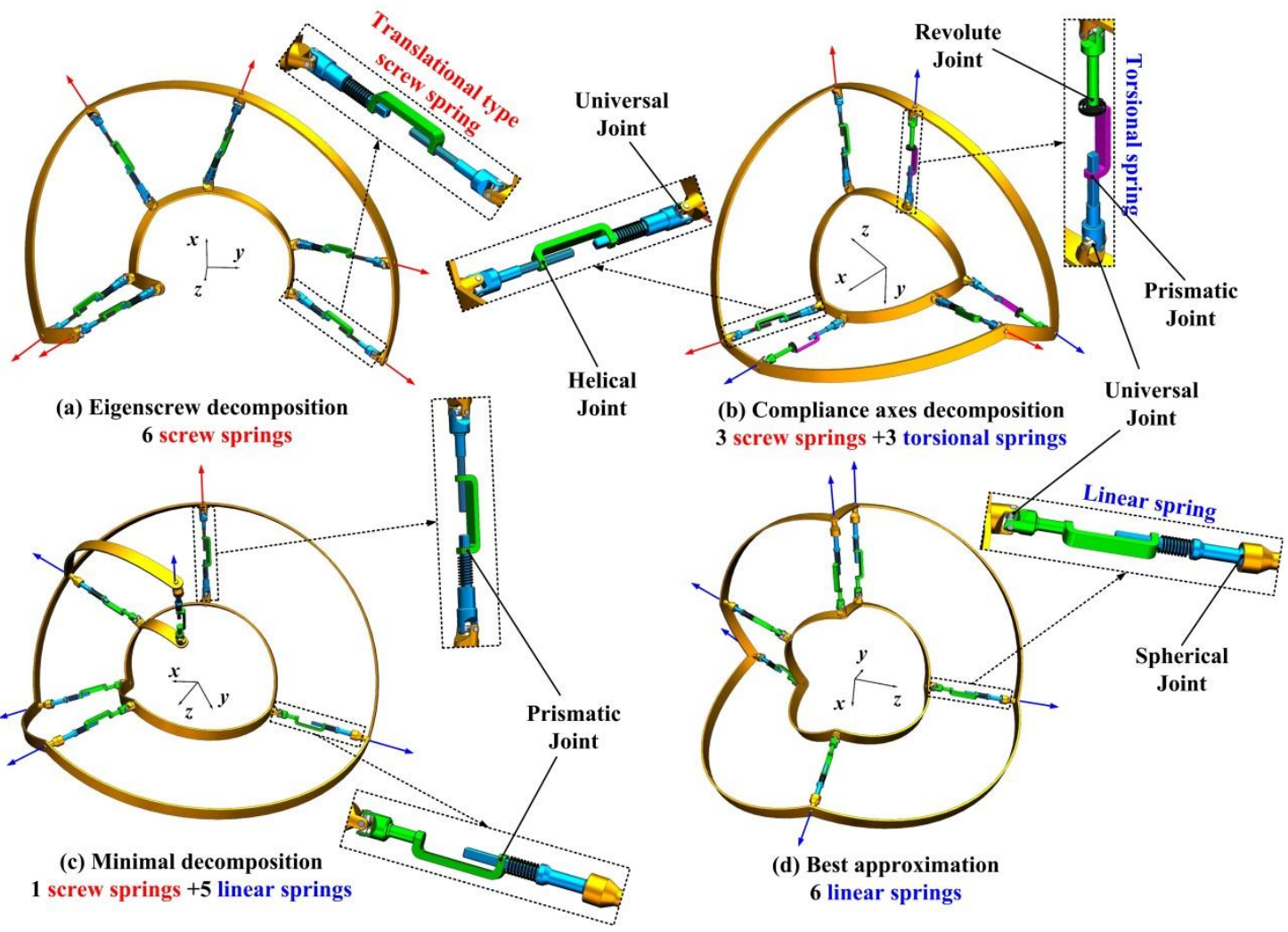

(d) Best approximation 6 linear springs

Fig.5 The physical implementation of the stiffness matrix. (a) Eigenscrew decomposition (b) Compliance axes decomposition (c) Minimal decomposition (d) Best approximation.

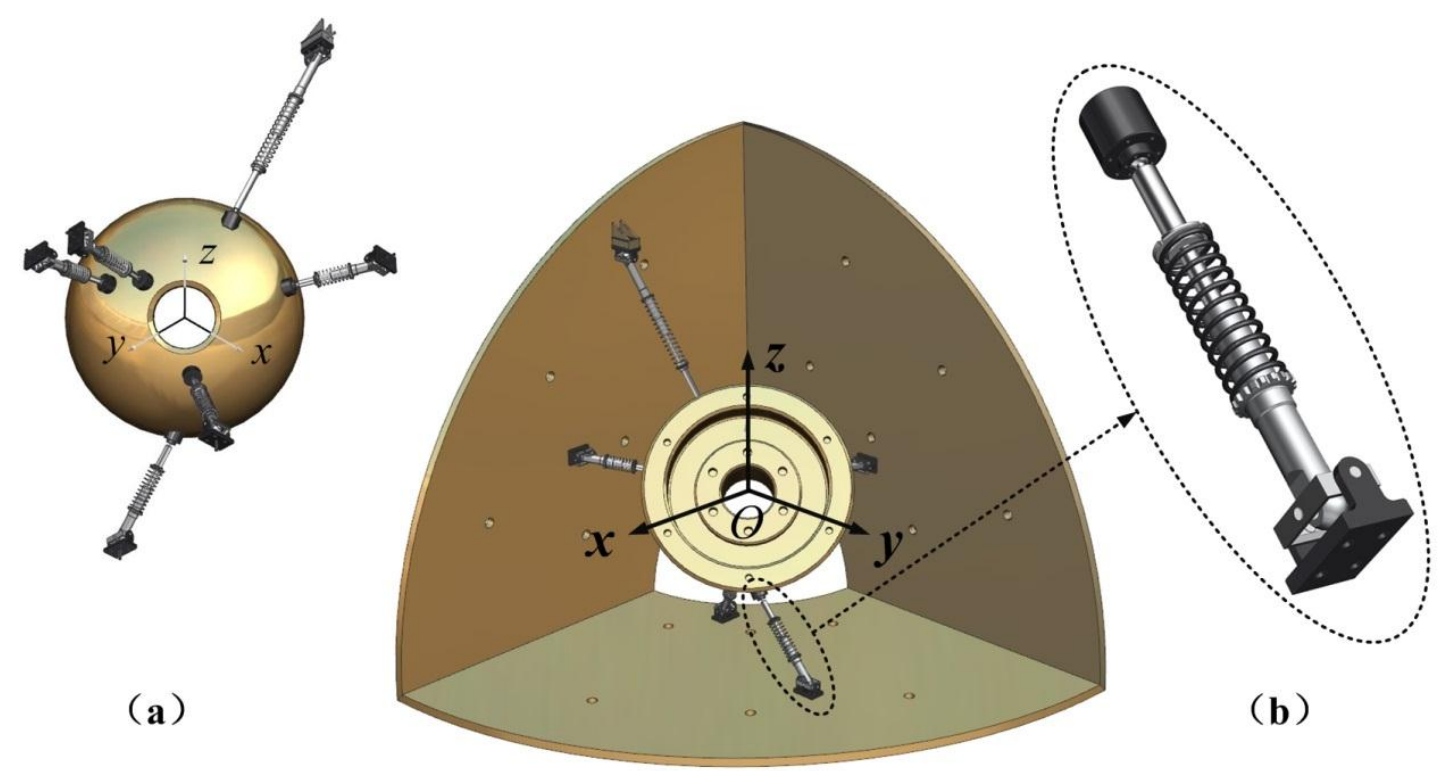


Fig.6 The passive compliance apparatus designed by the best approximation.

(a) The rear view of the apparatus without the base. (b) The realization of the simple spring.

Table.2 The realization of the desired stiffness matrix $\mathbf{K}$ and best-approximate stiffness matrix $\tilde{\mathbf{K}}$. $\mathbf{K}=\mathbf{w} \operatorname{diag}(\boldsymbol{k}) \mathbf{w}^{T}$, and $\tilde{\mathbf{K}}=\tilde{\mathbf{w}} \operatorname{diag}(\tilde{\boldsymbol{k}}) \tilde{\mathbf{w}}^{T}, \boldsymbol{h}$ denotes the pitches of the corresponding spring wrenches.

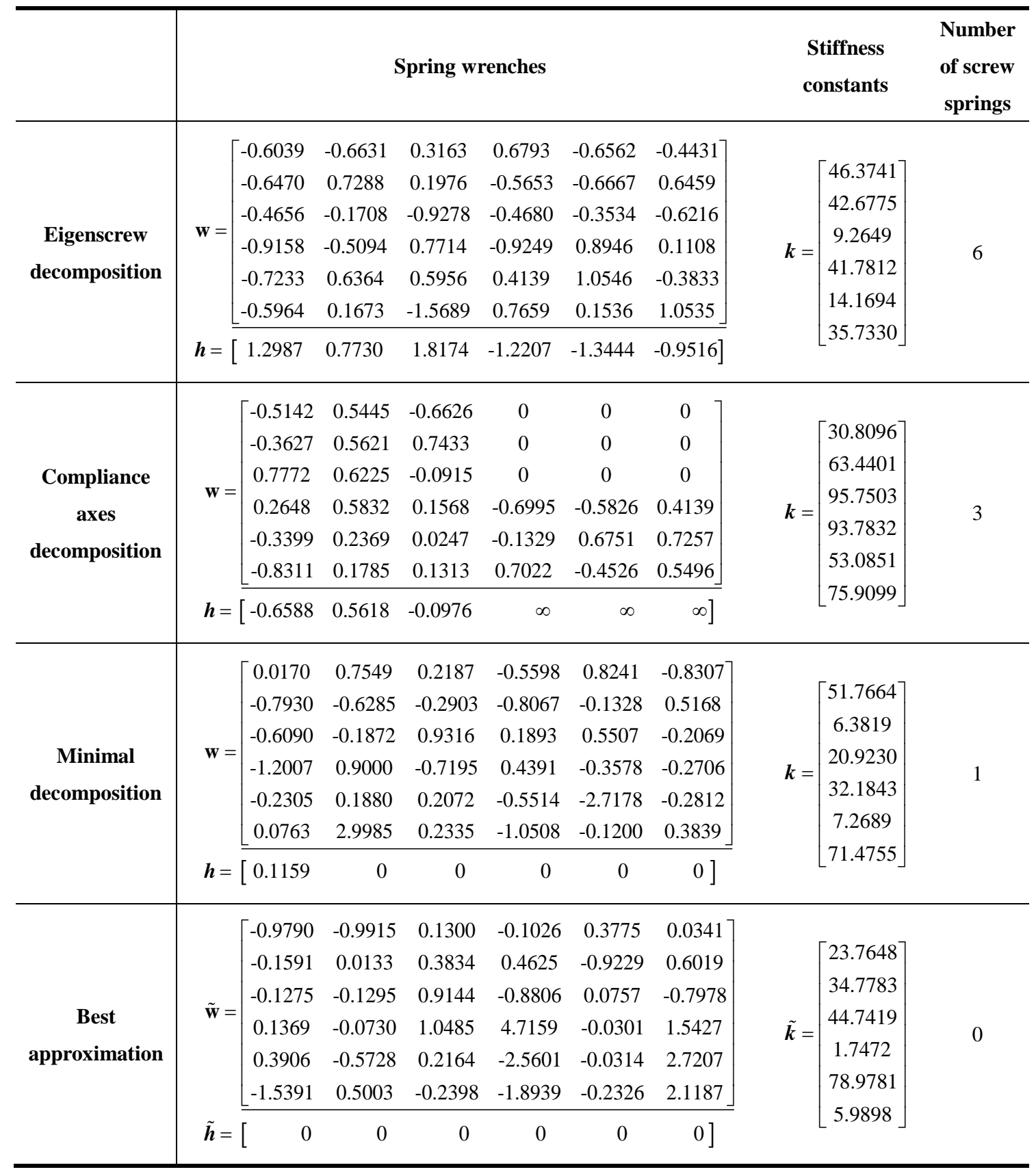

\subsection{The physical properties of the best-approximate stiffness matrix $\tilde{\mathbf{K}}$}

Section 3 has pointed out that the best approximation to stiffness matrix can retain the physical properties of the original stiffness matrix in many aspects. In this part, the verifications are presented combining with this numerical example. At first, the norm form is discussed. Converting the original stiffness matrix $\mathbf{K}$ into a special coordinate frame, the new coupling aspect 
$\underline{\mathbf{B}}=\mathbf{R}^{T} \mathbf{A} \hat{\mathbf{r}} \mathbf{R}+\mathbf{R}^{T} \mathbf{B R}$ becomes a diagonal matrix. In terms of the reference [20], the coordinates of origin of the special frame can be calculated. That is:

$$
\boldsymbol{r}=\left[\begin{array}{lll}
0.1663 & 0.1122 & -0.0940
\end{array}\right]^{T}
$$

This point is also the center of stiffness of $\mathbf{K}$. In addition, the rotational matrix $\mathbf{R}$ determining the directions of the special frame relative to the global frame can also be obtained as:

$$
\mathbf{R}=\left[\begin{array}{ccc}
-0.6566 & -0.5827 & 0.4789 \\
-0.6455 & 0.1057 & -0.7564 \\
-0.3901 & 0.8058 & 0.4455
\end{array}\right]
$$

Finally, the special coordinate frame $\{\underline{Q}\}$ in which the stiffness matrix $\mathbf{K}$ is turned into its norm form is identified, as shown in Fig.7. Combining with the congruence transformation of stiffness matrix, the norm form of $\mathbf{K}$ is obtained as:

$$
\underline{\mathbf{K}}=\operatorname{Ad}_{\mathbf{g}}^{T} \mathbf{K A d}_{\mathbf{g}}=\left[\begin{array}{cccccc}
61.090 & -7.890 & -3.013 & 40.317 & 0 & 0 \\
-7.890 & 42.672 & -22.467 & 0 & -28.404 & 0 \\
-3.013 & -22.467 & 86.238 & 0 & 0 & -5.913 \\
40.317 & 0 & 0 & 104.454 & -0.059 & 2.783 \\
0 & -28.404 & 0 & -0.059 & 114.983 & 3.641 \\
0 & 0 & -5.913 & 2.783 & 3.641 & 54.067
\end{array}\right]
$$

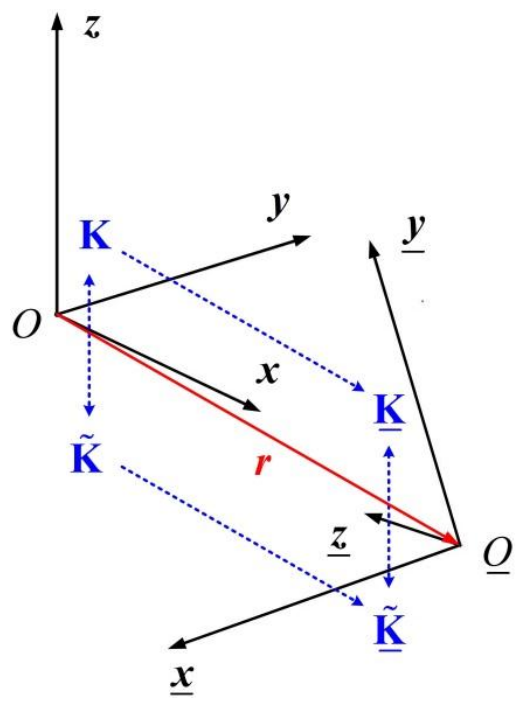

Fig.7 The norm forms of $\mathbf{K}$ and $\tilde{\mathbf{K}}$.

Next, to transform the $\tilde{\mathbf{K}}$ into the frame $\{\underline{O}\}$, it is interesting to find that: 


$$
\underline{\tilde{\mathbf{K}}}=\operatorname{Ad}_{\mathbf{g}}{ }^{T} \tilde{\mathbf{K}} \mathrm{Ad}_{\mathrm{g}}=\left[\begin{array}{cccccc}
61.090 & -7.890 & -3.013 & 38.317 & 0 & 0 \\
-7.890 & 42.672 & -22.467 & 0 & -30.404 & 0 \\
-3.013 & -22.467 & 86.238 & 0 & 0 & -7.913 \\
38.317 & 0 & 0 & 104.451 & -0.059 & 2.783 \\
0 & -30.404 & 0 & -0.059 & 114.983 & 3.641 \\
0 & 0 & -7.913 & 2.783 & 3.641 & 54.067
\end{array}\right]=\underline{\mathbf{K}}-\underline{\mu} \boldsymbol{\Delta}
$$

Note that the $\underline{\tilde{\mathbf{K}}}$ has three meanings. The basic meaning of $\underline{\tilde{\mathbf{K}}}$ is the representation of $\tilde{\mathbf{K}}$ in the particular frame $\{\underline{O}\}$. From the formula (33), it can be seen that the coupling aspect of $\underline{\mathbf{K}}$ is diagonal, which means $\underline{\tilde{\mathbf{K}}}$ is also the norm form of $\tilde{\mathbf{K}}$, and the best approximation $(\mathbf{K} \rightarrow \tilde{\mathbf{K}})$ does not change the center of stiffness of the original stiffness matrix. On the other hand, the formula (33) also indicates that $\underline{\tilde{\mathbf{K}}}$ is just the best approximation to $\underline{\mathbf{K}}$. That is to say, the best approximation keeps the same form of congruence transformation, and is independent with the choice of reference coordinate frame.

Thereafter, the compliance axes are studied. According to the eigenwrenches and eigentwists, the directions and positions of the wrench and twist compliance axes can be all determined. The results are listed in Table.3. Fig. 8 further exhibits the compliance axes in the global coordinate frame, in which the red vectors represent the compliance axes of $\mathbf{K}$ and the blue vectors represent the compliance axes of $\tilde{\mathbf{K}} . \boldsymbol{r}_{i}^{w}$ and $\boldsymbol{r}_{i}^{t}$ denote the coordinates of one point on the $i^{\text {th }}$ wrench compliance axis and the $i^{\text {th }}$ twist compliance axis, respectively. It can be verified that the $\boldsymbol{r}_{i}^{w}$ and $\boldsymbol{r}_{i}^{t}$ here are perpendicular to the corresponding wrench and twist compliance axis.

Table. 3 The directions and positions of the wrench/twist compliance axes of $\mathbf{K}$ and $\tilde{\mathbf{K}}$.

\begin{tabular}{|c|c|c|c|c|c|c|c|}
\hline & & \multicolumn{3}{|c|}{ Directions of the axes } & \multicolumn{3}{|c|}{ Positions of the axes } \\
\hline \multirow{6}{*}{$\mathbf{K}$} & wrench & $\lceil-0.5142]$ & $\lceil 0.5445\rceil$ & {$[-0.6626]$} & {$[0.5656]$} & {$[-0.0471]$} & {$[0.0999]$} \\
\hline & compliance & -0.3627 & 0.5621 & 0.7433 & -0.2215 & 0.2659 & 0.0727 \\
\hline & axes & 0.7772 & 0.6225 & {$[-0.0915]$} & 0.2708 & -0.1988 & -0.1329 \\
\hline & & 0.5826 & {$[-0.6995]$} & $0.4139]$ & 0.2155 & $-0.0510]$ & 0.4538 \\
\hline & twist com- & -0.6751 & -0.1329 & 0.7257 & 0.0686 & 0.3779 & -0.3295 \\
\hline & pliance axes & 0.4526 & 0.7022 & 0.5496 & $-0.1751]$ & 0.0208 & 0.0933 \\
\hline \multirow{3}{*}{$\tilde{\mathbf{K}}$} & wrench & {$[-0.5142]$} & 0.5445 & {$[-0.6626]$} & 0.5656 & -0.0471 & 0.0999 \\
\hline & compliance & -0.3627 & 0.5621 & 0.7433 & -0.2215 & 0.2659 & 0.0727 \\
\hline & axes & 0.7772 & 0.6225 & {$[-0.0915]$} & 0.2708 & {$[-0.1988]$} & {$[-0.1329]$} \\
\hline
\end{tabular}




\begin{tabular}{|c|c|c|c|c|c|c|}
\hline $\begin{array}{l}\text { twist com- } \\
\text { pliance axes }\end{array}$ & {$\left[\begin{array}{c}0.5721 \\
-0.6802 \\
0.4583\end{array}\right]$} & {$\left[\begin{array}{c}-0.7522 \\
-0.2123 \\
0.6238\end{array}\right]$} & {$\left[\begin{array}{l}0.3270 \\
0.7016 \\
0.6331\end{array}\right]$} & {$\left[\begin{array}{c}0.2045 \\
0.0523 \\
-0.1776\end{array}\right]$} & {$\left[\begin{array}{c}-0.1578 \\
0.4653 \\
-0.0320\end{array}\right]$} & {$\left[\begin{array}{c}0.5717 \\
-0.4006 \\
0.1486\end{array}\right]$} \\
\hline
\end{tabular}

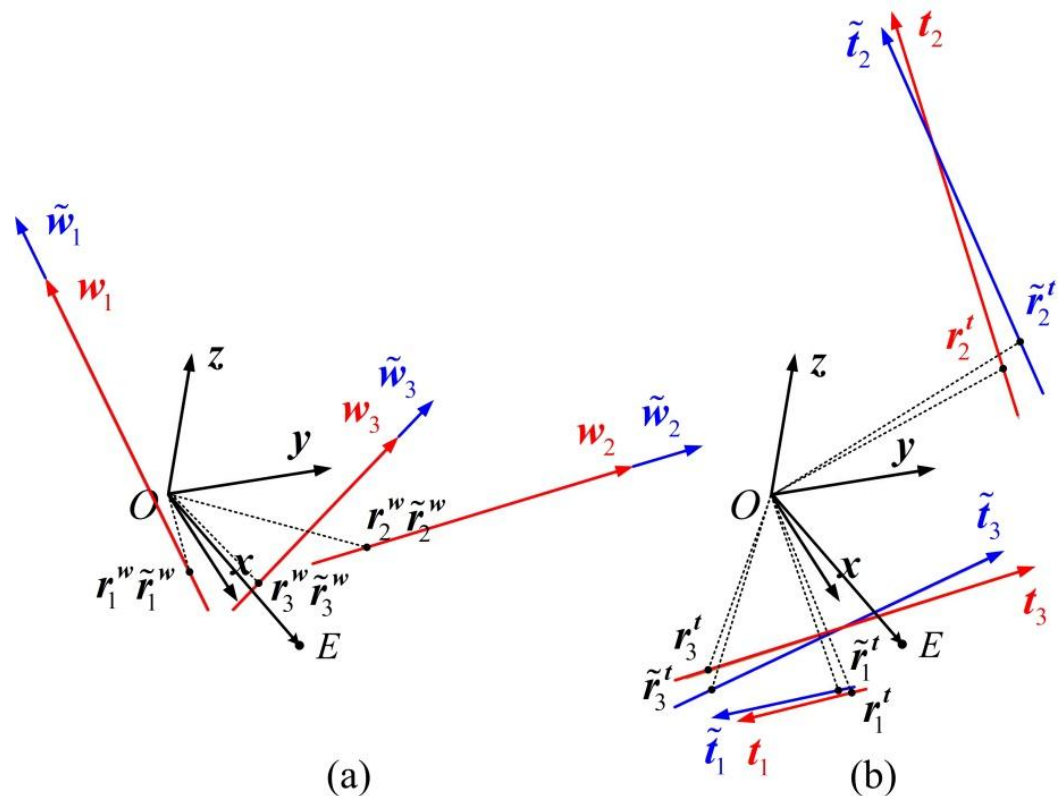

Fig.8 The compliance axes of $\mathbf{K}$ and $\tilde{\mathbf{K}}$ (a) Wrench compliance axes (b) Twist compliance axes.

It is clear that the wrench compliance axes of the two stiffness matrices are coincident. On the other hand, both the directions and positions of the twist compliance axes are changed after the best approximation. However, it is intuitively plausible that the differences between the two groups of twist compliance axes are not very large. After the recognition of the wrench/twist compliance axes, the center of elasticity can be identified. The coordinates of this point is equal to one-half the sum of the perpendicular vectors from the origin of global frame to the eigenwrenches or eigentwists [9]. Therefore, the location of the center of elasticity can be calculated as:

$$
\boldsymbol{r}^{E}=\frac{1}{2} \sum_{i=1}^{3} \boldsymbol{r}_{i}^{w}=\frac{1}{2} \sum_{i=1}^{3} \boldsymbol{r}_{i}^{t}=\frac{1}{2} \sum_{i=1}^{3} \tilde{\boldsymbol{r}}_{i}^{w}=\frac{1}{2} \sum_{i=1}^{3} \tilde{\boldsymbol{r}}_{i}^{t}=\left[\begin{array}{lll}
0.3092 & 0.0585 & -0.0305
\end{array}\right]^{T}
$$

The results show that, the best approximation to stiffness matrix does not lead to a new center of elasticity, and the point $E$ in Fig.8 is just the center of elasticity of both $\mathbf{K}$ and $\tilde{\mathbf{K}}$.

At last, the TSV and RSV of the original stiffness matrix and the best-approximate stiffness matrix are compared. The values are related to the deflections of the suspended rigid-body. It is unpractical to calculate the stiffness values with respect to all the deflections. For this reason, we only cite one translational deflection and one rotational deflection as examples. Without loss of generality, the two deflections are designed as follows: 


$$
\left.\boldsymbol{X}_{t r}=\left[\begin{array}{c}
3 / \sqrt{50} \\
4 / \sqrt{50} \\
5 / \sqrt{50} \\
0 \\
0 \\
0
\end{array}\right] \quad \boldsymbol{X}_{r o t}=\left[\begin{array}{c}
1 \\
1 \\
0
\end{array}\right) \times\left(\begin{array}{c}
3 / \sqrt{50} \\
4 / \sqrt{50} \\
5 / \sqrt{50}
\end{array}\right)\right]
$$

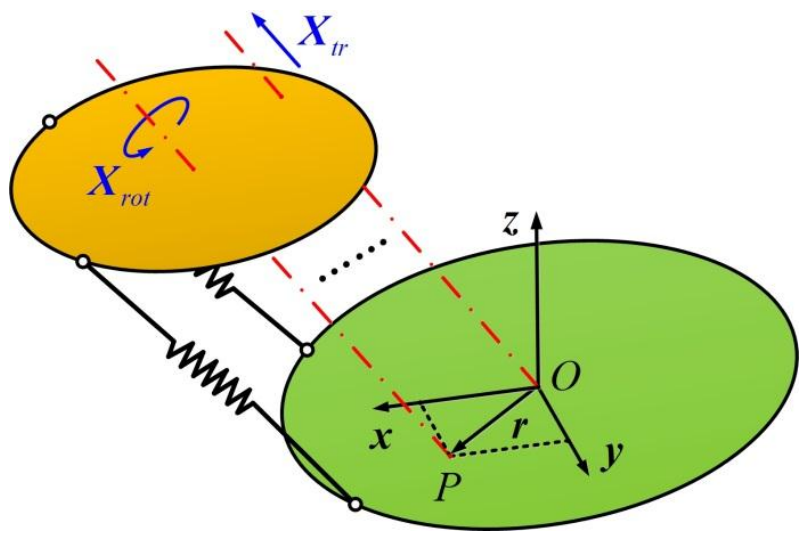

Fig.9 The translational deflection $\quad \boldsymbol{X}_{t r}$ and the rotational deflection $\boldsymbol{X}_{r o t}$.

The directions of the deflections are not along the axes of global coordinate frame, and the rotation axis does not pass though the origin, as shown in Fig.9. It is easy to calculate that:

$$
k_{t r}=\tilde{k}_{t r}=63.100 \quad k_{\text {rot }}=\tilde{k}_{r o t}=177.160
$$

Obviously, both the TSV and the RSV are equivalent between the original stiffness matrix and the best-approximate stiffness matrix. The same results can be derived for any other translational and rotational deflections.

\section{Conclusions}

In this paper, the best approximation to stiffness matrix is put forward for simplifying the realization of the non-isotropic stiffness matrices by purely simple springs connected in parallel. From the point of view in mathematics, the best-approximate stiffness matrix is the same to the original stiffness matrix except the diagonal entries of the off-diagonal blocks. Moreover, it has been proved that the best approximation is independent with the choice of reference coordinate frame, and the center of stiffness is invariant after the best approximation. The studies on the compliance axes also indicate that the best approximation keeps the direction and position of the wrench compliance axis unchanged, in the meanwhile, changes the twist compliance axis a little. By analyzing 
the TSV and RSV, the mechanical characteristics caused by an arbitrary translational or rotational deflection between the original stiffness matrix and the best-approximate stiffness matrix are proved to be the same.

In summary, the best approximation can reduce the changes of the original stiffness properties to a very great extent, and at the same time, simplify the physical implementation of the non-isotropic stiffness requirements significantly.

\section{Acknowledgments}

The work is supported by grants from the National Science Foundation of China (NSFC) under Grant 51305256, the National Basic Research Program of China (Grant No.2014CB046600), the National Science and Technology Major Project (Grant No.2014ZX04001-081-08), and the SJTU SMC-Morningstar Young Scholars program. The authors are grateful for these financial supports.

\section{References}

[1] S. Huang, The analysis and synthesis of spatial compliance, Marquette University 1998, (Ph.D. Thesis).

[2] D. E. Whitney, Quasi-static assembly of compliantly supported rigid parts, J. Dyn. Sys. Meas. Control 104(1) (1982) 65-77.

[3] R. G. Roberts, Minimal realization of an arbitrary spatial stiffness matrix with a parallel connection of simple and complex springs, IEEE Trans. Robot. Automat. 16(5) (2000) 603-608.

[4] J. Loncaric, Geometrical analysis of compliant mechanisms in Robotics, Harvard University 1985, (Ph.D. Thesis).

[5] S. Huang, J. Schimmels, The bounds and realization of spatial stiffnesses achieved with simple springs connected in parallel, IEEE Trans. Robot. Automat. 14(3) (1998) 466-475.

[6] S. Huang, J. Schimmels, The eigenscrew decomposition of spatial stiffness matrices, IEEE Trans. Robot. Automat. 16(2) (2000) 146-156.

[7] T. Patterson, H. Lipkin, Structure of robot compliance, J. Mech. Design 115(3) (1993) 576-580.

[8] H. Lipkin, T. Patterson. Geometrical Properties of Modelled Robot Elasticity: Part I — Decomposition, ASME Design Technical Conference, 1992, pp. 179-185. 
[9] N. Ciblak, H. Lipkin, Centers of stiffness, compliance, and elasticity in the modelling of robotic systems, ASME Design Technical Conference, Minneapolis, 1994, pp. 185-195.

[10] R. G. Roberts, Minimal realization of a spatial stiffness matrix with simple springs connected in parallel, IEEE Trans. Robot. Automat. 15(5) (1999) 953-958.

[11] K. Choi, S. Jiang, Z. Li, Spatial stiffness realization with parallel springs using geometric parameters, IEEE Trans. Robot. Automat. 18(3) (2002) 274-284.

[12] M. B. Hong, Y. J. Choi, Screw system approach to physical realization of stiffness matrix with arbitrary rank, J. Mech. Robot. 1(2) (2009) 021007.

[13] S. Huang S, J. Schimmels, Achieving an arbitrary spatial stiffness with springs connected in parallel, J. Mech. Design 120(4) (1998) 520-526.

[14] Y. Li, Q. Xu, Stiffness analysis for a 3-PUU parallel kinematic machine, Mech. Mach. Theory 43(2) (2008) 186-200.

[15] M. Ceccarelli, G. Carbone, A stiffness analysis for CaPaMan (Cassino parallel manipulator), Mech. Mach. Theory 37(5) (2002) 427-439.

[16] Z. Gao, D. Zhang, X. Hu, et al., Design, analysis, and stiffness optimization of a three degree of freedom parallel manipulator, Robotica 28(03) (2010) 349-357.

[17] F. Xi, D. Zhang, C. M. Mechefske, et al., Global kinetostatic modelling of tripod-based parallel kinematic machine, Mech. Mach. Theory 39(4) (2004) 357-377.

[18] X. J. Liu, Z. L. Jin, F. Gao, Optimum design of 3-DOF spherical parallel manipulators with respect to the conditioning and stiffness indices, Mech. Mach. Theory 35(9) (2000) $1257-1267$.

[19] G. Carbone, M. Ceccarelli, Comparison of indices for stiffness performance evaluation, Frontiers of Mechanical Engineering in China 5(3) (2010) 270-278.

[20] J. Loncaric, Normal forms of stiffness and compliance matrices, IEEE Trans. Robot. Automat. 3(6) (1987) 567-572.

[21] H. Lipkin, T. Patterson. Geometrical Properties of Modelled Robot Elasticity: Part II—Center of Elasticity, ASME Design Technical Conferences, 1992, pp. 13-16.

[22] B. El-Khasawneh, P. M. Ferreira, Computation of stiffness and stiffness bounds for parallel link manipulators, Int. J. Mach. Tool. Manu. 39(2) (1999) 321-342.

[23] V. T. Portman, Stiffness Evaluation of Machines and Robots: Minimum Collinear Stiffness 
Value Approach, J. Mech. Robot. 3(1) (2011) 011015.

[24] M. Griffis, J. Duffy, Global stiffness modeling of a class of simple compliant couplings, Mech. Mach. Theory 28(2) (1993) 207-224.

[25] N. Ciblak, H. Lipkin, Asymmetric Cartesian stiffness for the modeling of compliant robotic systems, Proc. 23rd Biennial ASME Mechanisms Conference, Minneapolis, 1994.

[26] A. Pashkevich, A. Klimchik, D. Chablat, Enhanced stiffness modeling of manipulators with passive joints, Mech. Mach. Theory 46(5) (2011) 662-679.

[27] David C Lay, Linear algebra and its applications, $4^{\text {th }}$ ed. Addison Wesley, Boston, 2010.

[28] J. Yu, Y. Zhao, H. Wang, et al., Compliance analysis and synthesis of a serial manipulator with redundant DOFs used in Tokamak, in: Advances in Reconfigurable Mechanisms and Robots II, Springer, London, 2015, pp. 757-767.

[29] R. M. Murray, Z. Li, S. S. Sastry, A mathematical introduction to robotic manipulation, CRC Press, New York, 1994. 